Int. J. Dev. Biol. 50: 209-222 (2006)

doi: $10.1387 / \mathrm{ijdb} .052066 \mathrm{jd}$

\title{
An anisotropic-viscoplastic model of plant cell morphogenesis by tip growth
}

\author{
JACQUES DUMAIS*,1, SIDNEY L. SHAW², CHARLES R. STEELE', SHARON R. LONG ${ }^{2}$ and PETER M. RAY² \\ ${ }^{1}$ Department of Organismic and Evolutionary Biology, Harvard University, Cambridge MA, USA, ${ }^{2}$ Department of Biological Sciences and \\ ${ }^{3}$ Department of Mechanical Engineering, Stanford University, Stanford CA, USA
}

\begin{abstract}
Plant cell morphogenesis depends critically on two processes: the deposition of new wall material at the cell surface and the mechanical deformation of this material by the stresses resulting from the cell's turgor pressure. We developed a model of plant cell morphogenesis that is a first attempt at integrating these two processes. The model is based on the theories of thin shells and anisotropic viscoplasticity. It includes three sets of equations that give the connection between wall stresses, wall strains and cell geometry. We present an algorithm to solve these equations numerically. Application of this simulation approach to the morphogenesis of tipgrowing cells illustrates how the viscoplastic properties of the cell wall affect the shape of the cell at steady state. The same simulation approach was also used to reproduce morphogenetic transients such as the initiation of tip growth and other non-steady changes in cell shape. Finally, we show that the mechanical anisotropy built into the model is required to account for observed patterns of wall expansion in plant cells.
\end{abstract}

KEY WORDS: anisotropy, cell morphogenesis, tip growth, viscoplasticity, wall expansion

\section{Introduction}

Plant cells acquire specific shapes according to two major modes of morphogenesis called diffuse growth and tip growth. In diffuse growth, expansion of the cell surface is distributed uniformly over the whole cell (Castle, 1955; Green, 1963). This mode of morphogenesis is seen in most cells of multicellular plants and in many algae. In tip growth, cells form long cylinders capped by a prolate dome where surface expansion takes place (Haberlandt, 1887; Reinhardt, 1892; Castle, 1958; Chen, 1973; Hejnowicz et al., 1977; Shaw et al., 2000; von Dassow et al., 2001). Tip growth is seen in a wide range of cells including root hairs, fungal hyphae and pollen tubes (Heath, 1990).

Diffusely-growing and tip-growing cells are surrounded by a stiff wall that maintains cell shape. Consequently, plant cell growth and morphogenesis are possible only to the extent that cells can cause their surrounding wall to expand locally. Wall expansion requires two complementary processes: i) the addition of new wall material by secretion and synthesis at the cell membrane and ii) the mechanical deformation of the wall via the tensional stresses excerted by the internal turgor pressure of the cell. The integration of these two processes into a single model of cell morphogenesis is a daunting task although some observations suggest a possible starting point. First, in a wide range of cell types it is possible to stop cell enlargement while wall deposition is maintained for some time leading to local thickening of the cell wall (Kiermayer, 1964; Schröter and Sievers, 1971; Roy et al., 1999). This treatment shows that deposition of wall material alone is not sufficient to drive expansion of the cell surface. On the other hand, stretching of the cell surface by turgor pressure cannot proceed without addition of new wall material because the cell wall would quickly thin and rupture. Based on these observations we propose a model where secretion and wall synthesis lead to growth in thickness while mechanical deformation by turgor pressure leads to expansion of the cell surface (Fig. 1A).

Historically, the mechanical deformation of the cell wall has been described using viscoplasticity theory (Lockhart, 1965a,b; Probine, 1966; Green et al., 1971; Ortega, 1990). A viscoplastic material differs from a viscous fluid in that it deforms irreversibly only for stress values that are beyond a plastic yield stress $\left(\sigma_{y}\right)$. Below the yield stress, the material deforms elastically but sustains no permanent deformation. For a stress greater than the yield stress, the rate of plastic deformation $(\dot{\epsilon})$ is proportional to the material extensibility $\Phi$ (an inverse viscosity) and to the applied stress $\sigma$ in excess of the yield stress. For uniaxial deformation, we write: $\dot{\epsilon}=\Phi\left(\sigma-\sigma_{y}\right), \sigma \geq \sigma_{y}$. An equation of this form was first proposed by Bingham (1922) to describe the flow of certain fluids such as paint and was later applied to wall extension by Lockhart

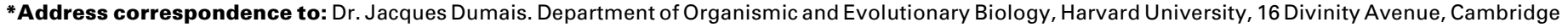
MA 02138, USA. Fax: +1-617-496-5854. e-mail: jdumais@oeb.harvard.edu
} 
A

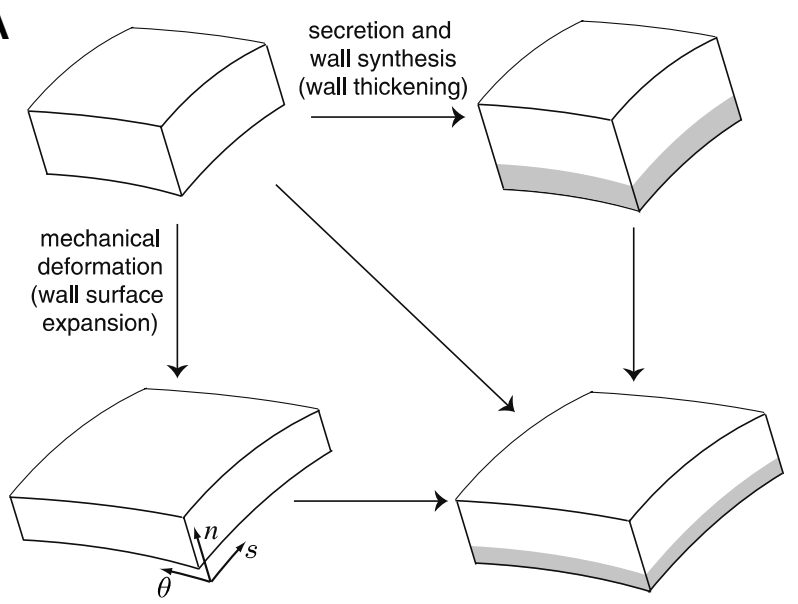

B

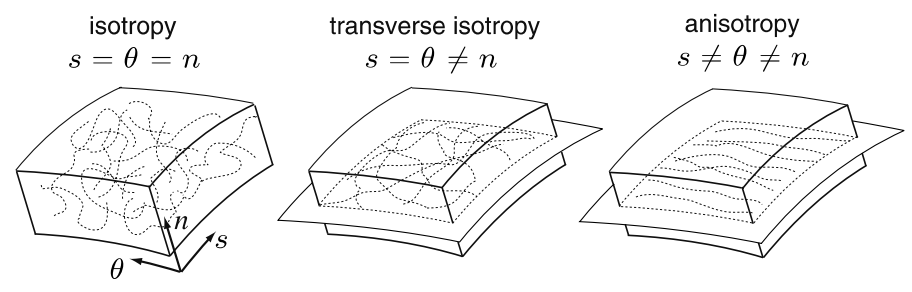

Fig. 1. Wall growth and structural anisotropy. (A) Two processes necessary for wall growth: mechanical deformation to increase the wall surface area and material deposition to preserve the thickness and integrity of the wall. (B) Three different types of wall architecture based on the orientation of cellulose microfibrils. Fully isotropic wall structures are not observed in nature. Transverse isotropy is typical of tip-growing cells and full anisotropy is typical of diffusely-growing cells. See Fig. 4 for a definition of the three principal axes.

(1965a,b). Since Lockhart, this simple equation has been used extensively to gain insights into the mechanics of cell growth. An important conclusion emerging from this work is that wall extensibility and its yield stress are not static mechanical properties (Green et al., 1971; Money and Harold, 1992); rather they are continually modulated by the cell both spatially and temporally.

Evidence for the viscoplastic model was obtained by manipulating turgor pressure in growing cells (Green etal., 1971; Passioura and Fry, 1992; Proseus et al., 1999). In this type of experiment, small increases and decreases in turgor pressure result in disproportionate increases and decreases in the rate of wall extension as would be expected if this rate is governed by a relation like Bingham's equation. In more extreme cases where turgor pressure is reduced to less than $30-50 \%$ of its normal value, cell expansion stops completely indicating that some threshold level of turgor pressure (yield stress) is necessary to drive wall extension (Cleland, 1959; Green et al., 1971; Cosgrove, 1985; Zhu and Boyer, 1992).

Although the viscoplastic model for wall extension is supported experimentally, it says little about the connection between wall assembly and wall mechanics. Numerous experiments have shown that the mechanical properties of the cell wall are, to a large extent, determined by the orientated deposition of cellulose microfibrils (Baskin, 2001). At least three broad types of cellulose organization must be considered (Fig. 1B). Cellulose microfibrils could be oriented randomly in all directions. The mechanical properties of the cell wall would then be isotropic. On the other hand, it is also possible for the cell wall to be organized into layers with randomly arranged cellulose microfibrils within each of these layers (Fig. 1B). This is in fact the wall organization observed in tip-growing cells (Houwink and Roelofsen, 1954; Belford and Preston, 1961; Newcomb and Bonnett, 1965; Kataoka, 1982). For this wall organization we expect the mechanical properties in the plane of the wall to be isotropic but these properties would likely differ from those in the normal direction because no cellulose microfibrils are aligned in that direction. This condition is called transverse isotropy. Finally, it is possible for the cellulose microfibrils to be aligned preferentially in one direction and thus provide full mechanical anisotropy to the cell wall (Fig. 1B). This wall organization is observed in many diffusely-growing cells such as the Nitellainternodal cell (Green,1962; Probine, 1966; Métraux et al., 1980; Richmond et al., 1980).

The goal of this paper is to develop a new model of plant cell morphogenesis based on a generalized Bingham's equation that accounts for the mechanical anisotropy arising from the orientation of cellulose microfibrils in the wall. We illustrate the fundamental features of this model using numerical simulations and comparisons with data from the literature.

\section{Mechanics of plant cell morphogenesis}

The mechanical aspect of plant cell morphogenesis can be viewed as the deformation of a thin pressurized shell (see for example Martynov, 1975; Hejnowicz et al., 1977; Chaplain and Sleeman, 1990; Steele, 2000). A shell is considered thin if it has a small thickness compared to its other spatial dimensions (Flügge, 1973). This definition fits most growing plant cells which typically have a wall thickness of $0.1-0.5 \mu \mathrm{m}$ and radii of

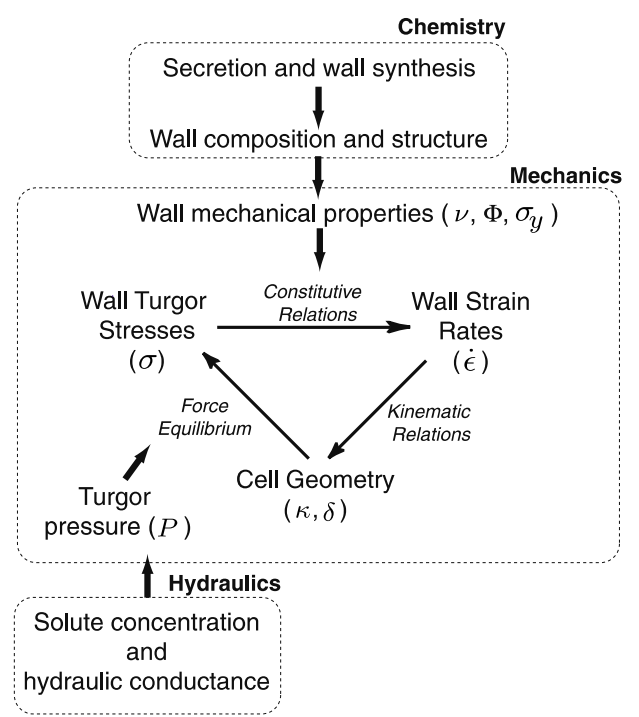

Fig. 2. Fundamental mechanical relations for cell morphogenesis. Cellular control of morphogenesis is possible via the wall mechanical properties and turgor pressure. 
curvature in excess of $5 \mu \mathrm{m}$.

Three types of variables must be considered in a description of cell morphogenesis using shell theory. These variables are the wall turgor stresses $(\sigma)$, the wall strain rates $(\dot{\epsilon})$ and geometrical variables such as the curvatures $(\kappa)$ of the cell surface and the cell wall thickness $(\delta)$. The connections between the stresses, strain rates and geometrical variables are given by three fundamental relations (Ugural, 1999) (Fig. 2). The constitutive relations describe the mechanical behavior of the material. Specifically for the cell wall, they give the surface strains in terms of the wall stresses and the wall mechanical properties. The kinematic equations give the deformation of the cell geometry that results from stretching of the cell surface. This set of equations is derived from geometrical considerations solely and does not require any assumption about the mechanism of surface extension. Finally, the equilibrium equations describe the force balance on a shell element. For a plant cell, there must be equilibrium between the normal force generated by turgor pressure and the wall tensions.

In considering the principles behind the morphogenesis of walled cells we make the following assumptions:

i) Axisymmetric cell geometry - We consider the cell geometry to be axisymmetric but otherwise free to change. The axisymmetric geometry is a good approximation for many plant cells and in particular for tip-growing cells (Fig. 3). Accordingly, stresses and strain rates are defined along three principal directions corresponding to the meridional (subscript $s$ ), circumferential (subscript $\theta$ ) and normal (subscript $n$ ) directions on the cell (Fig. 4). Furthermore, for an axisymmetric geometry, all variables and parameters such as the mechanical properties can be expected to vary solely with the meridional position $(s)$.

ii) The rate of wall deposition matches the rate of wall thinning due to in-plane expansion - Typically the thickness of the cell wall in the growth zone is roughly constant (e.g. Hejnowicz et al., 1977). We must thus conclude that the wall thinning resulting from expansion of the cell surface is closely matched by deposition of new wall material.

iii) Turgor pressure is supported by in-plane wall stresses Given that the cell wall is thin compared to the size of the cell and that the curvature of the cell surface does not change abruptly, it is fair to assume that turgor pressure is supported only by tensional forces in the plane of the wall while, relative to these, the normal (i.e., perpendicular to the cell wall surface) stress and bending stresses are negligible. In other words, we assume that the plant cell supports its internal turgor pressure in a way analogous to a rubber balloon or a soap bubble. Note that the absence of substantial stresses in the normal direction does not preclude strain in that direction.

The above assumptions fit a wide range of diffusely-growing and tip-growing cells. To these general assumptions we add one assumption that applies specifically to tip-growing cells.

iv) The cell wall is transversely isotropic- Although our model is derived for a fully anisotropic material, we want to consider closely its application to tip growth where the random organization of cellulose microfibrils in the plane of the cell wall suggests that the mechanical properties would be transversely isotropic (Fig. 1B). This conclusion is supported by recent observations made on tip-growing root hairs (Dumais et al., 2004).

\section{Constitutive relations}

In order to relate turgor stresses to the rates of wall extension we need some knowledge of the constitutive behavior of the wall material. Bingham's equation for a viscoplastic material under uniaxial stress was generalized by Hohenemser and Prager (1932), Prager $(1937,1961)$ and Oldroyd (1947) for a multiaxial state of stress. Our derivation follows that of Prager (1937) and standard treatments of plasticity (Hill, 1950). To represent the constitutive behavior of the cell wall under multiaxial stress, the uniaxial yield stress and flow are reformulated into a multiaxial yield criterion and a flow rule.

Yield criterion - For multiaxial stress, we need a measure of stress, $\sigma_{e}=f\left(\sigma_{s}, \sigma_{\theta}, \sigma_{n}\right)$, so that the cell wall yields for $\sigma_{e} \geq \sigma_{y}$. The function $\sigma_{e}$ is called the effective stress and the condition $\sigma_{e} \geq \sigma_{y}$ is the yield criterion. The turgor-induced stresses acting on a volume element of wall material can deform this element by dilatation (change in volume) and by distortion (shear strain). Dilatational stress tends to expand or compress the wall volume without causing distortion of the polymeric network, while distortional stress distorts the material without necessarily changing its volume. A general yield criterion can be formulated with these two components of stress (Christensen, 1997) although yield is often independent of moderate dilatation. For a polymeric material such as the plant cell wall, the distortional stress promotes the slippage of polymer chains that must occur during yielding. It is therefore

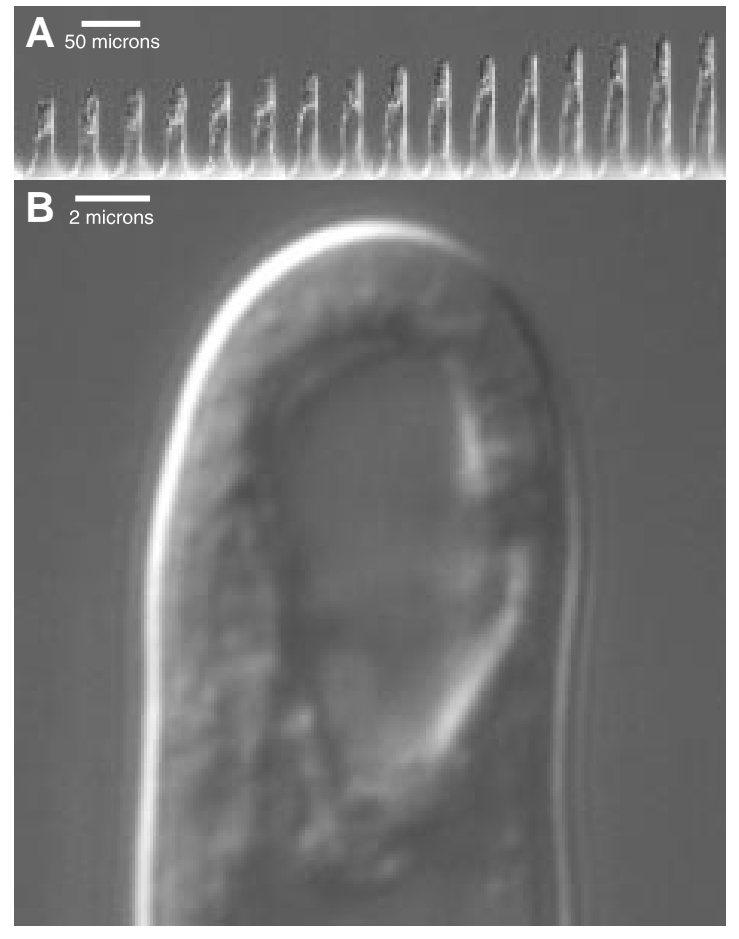

Fig. 3. Tip growth in root hairs. (A) Time-lapse images of an elongating root hair of Medicago truncatula. Images were taken every 10 minutes. Note the dense cytoplasm following precisely the advance of the tip. Surface expansion is high near the pole of the cell and decreases to nearly zero at the juncture between the dome and the cylindrical part of the cell. A slight circumferential creep seems to persist even far from the growing tip and this leads to a gradual widening of the cell axis. (B) Close-up of the growing tip. 
natural to define $\sigma_{e}$ only in terms of distortional stress. For an isotropic, incompressible material, a commonly used yield criterion is the von Mises criterion:

$\sigma_{e}=\frac{1}{\sqrt{2}}\left[\left(\sigma_{s}-\sigma_{\theta}\right)^{2}+\left(\sigma_{\theta}-\sigma_{n}\right)^{2}+\left(\sigma_{n}-\sigma_{s}\right)^{2}\right]^{1 / 2} \geq \sigma_{y}$

(Ugural and Fenster, 1975). Because stresses are present only as differences, the fraction of the total stress shared by all principal directions (i.e., the dilatational stress) does not contribute to the effective stress.

The energy that is stored in an elastic material that obeys Hooke's Law increases with the square of the imposed stresses (Ugural and Fenster, 1975). The sum of the squares of the differences in stress between the principal axes is thus an index for the total distortional strain energy that is stored elastically in the material. The von Mises yield criterion embodies, therefore, the principle that irreversible deformation occurs when the distortional energy in the material reaches a certain threshold value (indexed by $\sigma_{y}^{2}$ ).

Hill (1950) generalized the von Mises criterion to include materials with anisotropic yield stresses $\sigma_{y}^{(s)}, \sigma_{y}^{(\theta)}$ and $\sigma_{y}^{(n)}$ in the $s, \theta$ and $n$ directions. Hill's yield criterion is:

$\sigma_{e}=\left[A\left(\sigma_{s}-\sigma_{\theta}\right)^{2}+B\left(\sigma_{\theta}-\sigma_{n}\right)^{2}+C\left(\sigma_{n}-\sigma_{s}\right)^{2}\right]^{1 / 2} \geq \sigma_{y}$

Here, $\sigma_{y}$ is now a global yield stress to be defined in terms of the uniaxial yield stresses. Values of the coefficients $A, B$ and $C$ are found by evaluating the function for the three uniaxial states of stress (e.g., by substituting $\sigma_{s} \neq 0$ and $\sigma_{\theta}=\sigma_{n}=0$ and ensuring that the equation then reduces to $\sigma_{s} \geq \sigma_{y}^{(s)}$ ).

This gives: $A+C=\left(\sigma_{y} / \sigma_{y}^{(s)}\right)^{2}, A+B=\left(\sigma_{y} / \sigma_{y}^{(\theta)}\right)^{2}$ and $B+C=\left(\sigma_{y} / \sigma_{y}^{(n)}\right)^{2}$.

Solving for each coefficient, we find:

$$
\begin{aligned}
2 A & =\left(\frac{\sigma_{y}}{\sigma_{y}^{(s)}}\right)^{2}+\left(\frac{\sigma_{y}}{\sigma_{y}^{(\theta)}}\right)^{2}-\left(\frac{\sigma_{y}}{\sigma_{y}^{(n)}}\right)^{2} \\
2 B & =\left(\frac{\sigma_{y}}{\sigma_{y}^{(\theta)}}\right)^{2}+\left(\frac{\sigma_{y}}{\sigma_{y}^{(n)}}\right)^{2}-\left(\frac{\sigma_{y}}{\sigma_{y}^{(s)}}\right)^{2} \\
2 C & =\left(\frac{\sigma_{y}}{\sigma_{y}^{(n)}}\right)^{2}+\left(\frac{\sigma_{y}}{\sigma_{y}^{(s)}}\right)^{2}-\left(\frac{\sigma_{y}}{\sigma_{y}^{(\theta)}}\right)^{2}
\end{aligned}
$$

If the cell wall is fully isotropic then $A=B=C=1 / 2$ so that Hill's yield criterion reduces to the von Mises criterion. Hill's criterion extends to anisotropic materials the above-mentioned principle that a material yields when the stored distortional energy reaches a certain threshold value indexed by the square of the material's global yield stress, $\sigma_{y}$.

If Eq. (2) is substituted into Eq. (1) it will be seen that $\sigma_{y}$ is present on both sides of the inequality. It can be concluded that however $\sigma_{y}$ is defined it will not affect the yield criterion. In fact, $\sigma_{y}$ could be eliminated altogether from Eq. (1). However, we keep $\sigma_{y}$ in Eq. (1) to maintain a close parallel with Bingham's equation. A natural definition for $\sigma_{y}$ would be the mean of the three uniaxial yield stresses but here we follow previous usage (Capsoni et al., 2001) and define $\sigma_{y}$ solely in terms of the in-plane yield stresses,

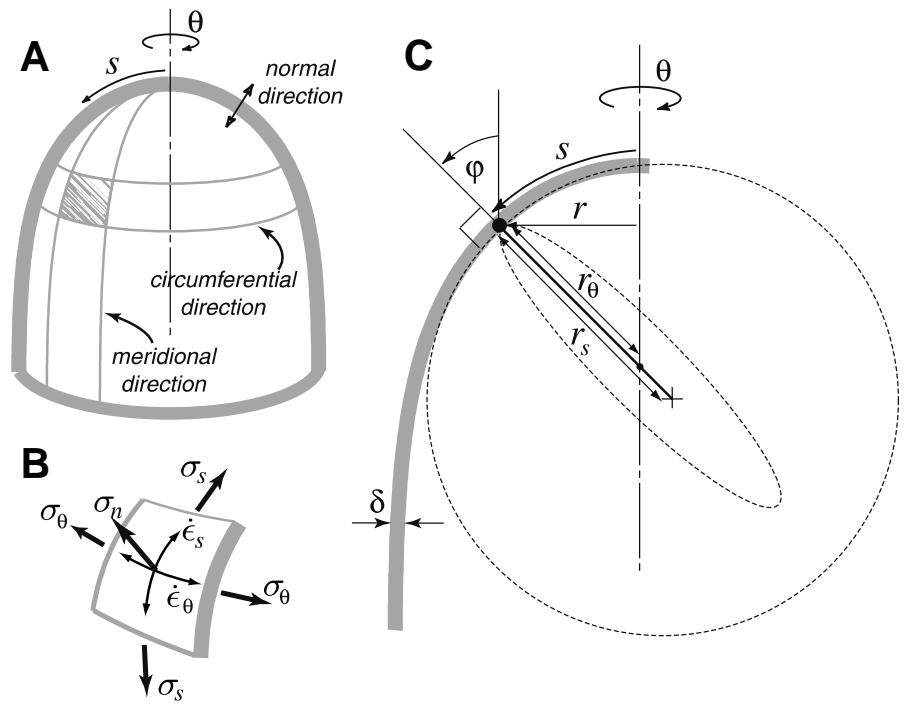

Fig. 4. Geometry of tip-growing cells. (A) Dome of a tip-growing cell showing a typical shell element (cross-hatched region) and the principal directions corresponding to the surface normal, the meridians and the parallels of the dome. (B) Principal stress $(\sigma)$ and strain rates $(\dot{\epsilon})$ acting on a shell element. The stress acting normal to the cell surface $\left(\sigma_{n}\right)$ is negligible compared to the stresses acting in the plane of the cell wall. (C) Geometry of a doubly-curved surface. The two principal curvatures at a point are illustrated as circles tangent to the surface. One circle, in face view, shows the first radius of curvature $\left(r_{s}=1 / \kappa_{s}\right)$; a second circle, in oblique view, shows the second radius of curvature $\left(r_{\theta}=1 / \kappa_{\theta}\right) . r$ is the radial distance from the axis of the cell and $\varphi$ is the angle between the normal to the surface and the axis of the cell.

that is, $\sigma_{y}=\left(\sigma_{y}^{(s)}+\sigma_{y}^{(\theta)}\right) / 2$. The in-plane yield stresses are the only yield stresses that are practical to measure experimentally and the final equations take a simpler form when this choice is made. We emphasize, however, that the conclusions of the paper are not affected by the definition used for $\sigma_{y}$.

A useful graphical representation of the yield criterion can be given for plane stress. Setting $\sigma_{n}=0$ in Eq. (1), squaring and expanding the terms in brackets reveal that Eq. (1) defines an elliptical yield locus: $(A+C) \sigma_{s}{ }^{2}+(A+B) \sigma_{\theta}{ }^{2}-2 A \sigma_{s} \sigma_{\theta}=\sigma_{y}{ }^{2}$ (Fig. 5A). The cell wall yields for stress combinations that fall on or outside the yield locus. The yield locus can be interpreted as the limit where the distortional elastic energy stored in the cell wall is such that any further increase therein leads to irreversible deformation.

Flow rule The flow rule specifies how the strain rate should be partitioned among the principal directions when the yield criterion is met. As stated in Bingham's equation, strain rate is expected to be proportional to the amount of stress in excess of the yield stress. For multiaxial stress, Prager (1937) defined this excess stress as the difference between the effective stress and the global yield stress, i.e., $\sigma_{e}-\sigma_{y}$. Flow should therefore be proportional to this excess stress. However, we face the additional complication of partitioning this flow between the three principal directions. Intuitively, one might expect that the flow will be such that it maximizes the dissipation of the distortional elastic energy stored in the cell wall. In other words, the relative distribution of 
flow between the three principal directions will be proportional to the contribution of each of these directions to the stored energy. Let $H=\sigma_{e}^{2} / 2$ represent the stored distortional elastic energy. In the theory of plasticity, $H$ is called the flow potential (Hill, 1950). The direction of greatest dissipation is given by the vector $\nabla H=\partial H / \partial \sigma_{s} \vec{e}_{s}+\partial H / \partial \sigma_{\theta} \vec{e}_{\theta}+\partial H / \partial \sigma_{n} \vec{e}_{n}$, where $\vec{e}_{i}$ is a unit vector in the $i$ direction. The components of $\nabla H$ indicate how the flow should be partitioned between the principal directions. Since we are looking only for fractional coefficients to multiply the excess stress, this vector should be first normalized by dividing by its length $K=\|\nabla H\|=\left[\sum_{i}\left(\partial H / \partial \sigma_{i}\right)^{2}\right]^{1 / 2}$. Graphically, the gradient of the flow potential defines vectors perpendicular to the yield locus (Fig. 5).

If, as indicated above, the strain rate in each of the directions $s, \theta$ and $n$ is proportional to $\left(\sigma_{e}-\sigma_{y}\right)$ and strain is partitioned among these directions in proportion to their contribution to stored distortional elastic energy, then we can write the following basic constitutive equation:

$$
\dot{\epsilon}_{i}=\Phi\left(\sigma_{e}-\sigma_{y}\right) \frac{1}{K} \frac{\partial H}{\partial \sigma_{i}}, \text { for } i=s, \theta, n \text { and } \sigma_{e} \geq \sigma_{y}
$$

where the coefficient $\Phi$ is the cell wall extensibility (the reciprocal of viscosity). Equation (3) is the constitutive equation adopted by Prager (1937) for a viscoplastic material under multiaxial stress. Its similarity with Bingham's equation is obvious. By differentiating $H$, we can write the strain rate ratios explicitly in terms of stresses and mechanical properties:

$$
\begin{aligned}
& \dot{\epsilon}_{s}: \dot{\epsilon}_{\theta}: \dot{\epsilon}_{n}=\frac{1}{K} \frac{\partial H}{\partial \sigma_{s}}: \frac{1}{K} \frac{\partial H}{\partial \sigma_{\theta}}: \frac{1}{K} \frac{\partial H}{\partial \sigma_{n}}= \\
& \frac{(A+C) \sigma_{s}-A \sigma_{\theta}}{K}: \frac{(A+B) \sigma_{\theta}-A \sigma_{s}}{K}: \frac{-C \sigma_{s}-B \sigma_{\theta}}{K}
\end{aligned}
$$

where

$$
K=\left[2\left(A^{2}+A C+C^{2}\right) \sigma_{s}{ }^{2}+2\left(A^{2}+A B+B^{2}\right) \sigma_{\theta}{ }^{2}+2\left(-2 A^{2}-A B-A C+C B\right) \sigma_{s} \sigma_{\theta}\right]^{1 / 2}
$$

and $\sigma_{n}$ was set to zero (plane stress) after taking the derivatives. From Eq. (4) and keeping in mind Eq. (2), we see that a relatively high stress or low yield stress in a given direction will give a relatively high strain rate in that direction. Substituting Eq. (4) into Eq. (3), we get constitutive equations for a general anisotropic material under plane stress. Note that in Eq. (3) the sum of the strain rates is zero as can be seen by adding the three directional terms of Eq. (4). This indicates that the volume of the deforming cell wall is constant. On the other hand, the absolute rate of deformation, $\left(\sum_{i} \dot{\epsilon}_{i}^{2}\right)^{1 / 2}$, is equal to $\Phi\left(\sigma_{e}-\sigma_{y}\right)$. Thus our model, like Bingham's equation, has an absolute rate of deformation that is a linear function of the applied stress.

If we now assume that the material is transversely isotropic $\left(\sigma_{y}^{(s)}=\sigma_{y}^{(\theta)}\right)$ and use the above definition for the global yield stress, we find $\sigma_{y}=\left(\sigma_{y}^{(s)}+\sigma_{y}^{(\theta)}\right) / 2=\sigma_{y}^{(s)}=\sigma_{y}^{(\theta)}$. Substituting in Eq. (2), we get $A=1-\frac{1}{2}\left(\sigma_{y} / \sigma_{y}^{(n)}\right)^{2}$ and $B=C=1-A$. The role played by the parameter $A$ in the viscoplastic equations is analogous to that of the Poisson's ratio $(\nu)$ in linear elasticity. We emphasize this similarity by substituting the symbol $\nu$ in place of $A$ and calling this new parameter the flow coupling. The flow coupling $\nu$ is a dimensionless mechanical property affecting the distribution of strain between the principal directions. For most materials, $\nu$ ranges between 0 and 1 .

Expanding Eq. (3) for plane stress and transverse isotropy, we obtain the following set of constitutive equations for the cell wall:

A

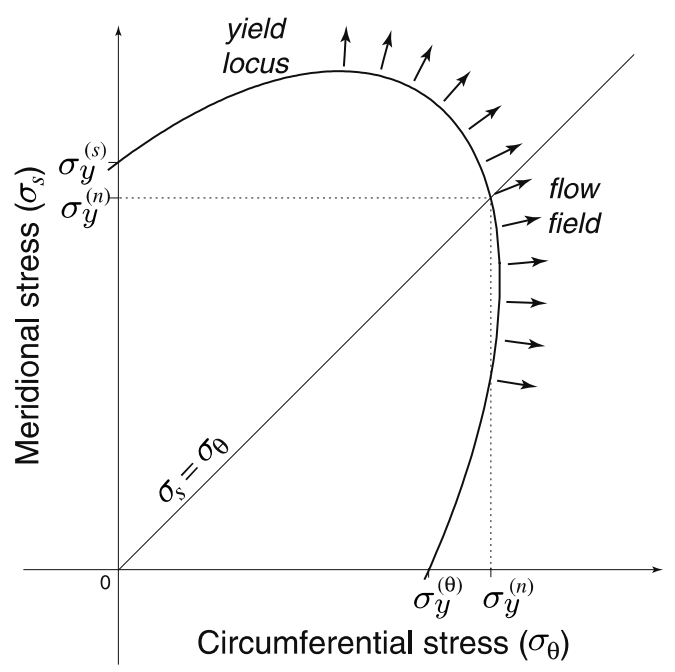

B

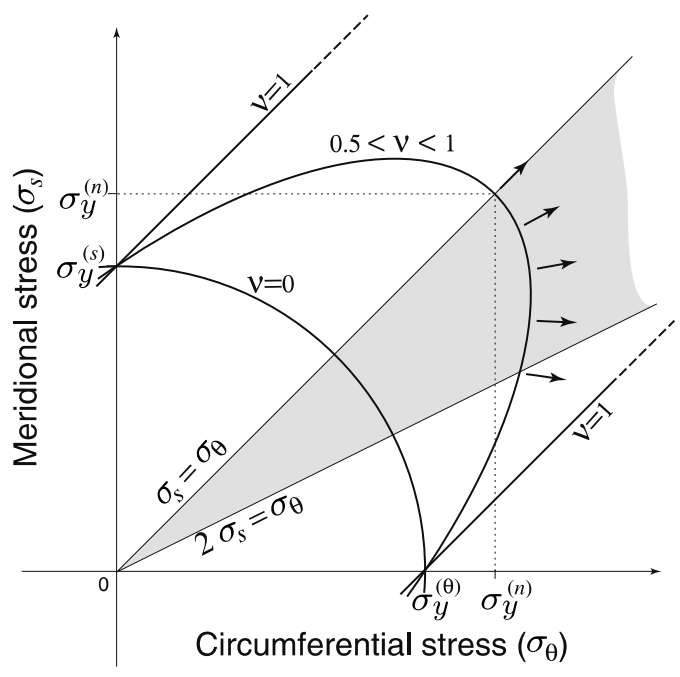

Fig. 5. Yield locus for a viscoplastic material. (A) Yield locus and flow field for an anisotropic material $\left(\sigma_{y}^{(s)}>\sigma_{y}^{(n)}>\sigma_{y}^{(\theta)}\right)$. The wall flows irreversibly for stress combinations that fall on or outside the yield locus. Arrows indicate the direction of flow. (B) Three possible yield loci for a transversely isotropic material. The loci corresponding to $\nu=0$ and $\nu=1$ represent two extreme cases. The locus $0.5<\nu<1$ is achieved if $\sigma_{y}^{(n)}>\sigma_{y}^{(\theta)}=\sigma_{y}^{(s)}$. A possible flow field associated with this yield locus is superposed on the region of likely stress combinations for a tipgrowing cell (shaded area). 


$$
\left.\begin{array}{l}
\dot{\epsilon}_{s}=\Phi\left(\sigma_{e}-\sigma_{y}\right)\left(\frac{\sigma_{s}-\nu \sigma_{\theta}}{K}\right) \\
\dot{\epsilon}_{\theta}=\Phi\left(\sigma_{e}-\sigma_{y}\right)\left(\frac{\sigma_{\theta}-\nu \sigma_{s}}{K}\right) \\
\dot{\epsilon}_{n}=\Phi\left(\sigma_{e}-\sigma_{y}\right)\left(\frac{(\nu-1)\left(\sigma_{s}+\sigma_{\theta}\right)}{K}\right)
\end{array}\right\} \text { for } \sigma_{e} \geq \sigma_{y}
$$

where, $\quad K=\left[\beta \sigma_{s}^{2}+\beta \sigma_{\theta}^{2}+(\beta-6 \nu) \sigma_{s} \sigma_{\theta}\right]^{1 / 2} \quad$ and $\beta=2 \nu^{2}-2 \nu+2$. Note that for $\sigma_{e}<\sigma_{y}$ the strain rates are zero. The normal strain rate $\left(\dot{\epsilon}_{n}\right)$ will in general be negative since by definition $\nu \leq 1$. The third equation thus gives the thinning of the wall that would occur due to stretching if new wall material were not being concomitantly deposited to maintain wall thickness. It should also be emphasized that all variables in Eq. (5), including the mechanical properties- $\Phi, \sigma_{y}$ and $\nu$, are functions of the meridional position $(s)$.

\section{Kinematic relations}

Kinematic relations for axisymmetric shells are well known and were discussed in the context of plant cell morphogenesis in several papers (Hejnowicz and Sievers, 1971; da Riva Ricci and Kendrick, 1972; Hart and Trainor, 1989; Pelcé and Pocheau, 1992; and others). Consider the displacement of any material point on the surface of a growing tip. As the cell elongates, the material point will be displaced along a curved trajectory until it becomes part of the non-growing region of the cell (Fig. 6). Our equations are based on the velocity vector $(\vec{V})$ of such material points. The velocity vector can be decomposed into components normal $\left(v_{n}\right)$ and tangential $\left(v_{t}\right)$ to the cell surface. The meridional and circumferential strain rates are defined in terms of the velocity components as follows (see Appendix A):

$$
\begin{aligned}
& \dot{\epsilon}_{s}=v_{n} \kappa_{s}+\frac{\partial v_{t}}{\partial s} \\
& \dot{\epsilon}_{\theta}=v_{n} \kappa_{\theta}+\frac{v_{t} \cos \varphi}{r}
\end{aligned}
$$

In these equations, the strain rates $\left(\dot{\epsilon}_{s}, \dot{\epsilon}_{\theta}\right)$ are related to the displacement velocities of the cell surface $\left(v_{n}, v_{t}\right)$ using parameters that describe the local surface geometry, namely, the curvatures $\left(\kappa_{s}\right.$ and $\kappa_{\theta}$, the reciprocals of the respective radii of curvature), the angle between the normal to the surface and the cell axis $(\varphi)$, the cross-sectional radius $(r)$ and the meridional distance $(s)$ (Fig. 4). Here, the normal strain rate $\left(\dot{\epsilon}_{n}\right)$ is equal to the thinning due to expansion in the plane (as in Eq. (5)) combined with the relative rate of thickening due to deposition of new wall material:

$$
\dot{\epsilon}_{n}=-\left(\dot{\epsilon}_{s}+\dot{\epsilon}_{\theta}\right)+\frac{D}{\delta}=0
$$

where $D$ denotes the rate of wall deposition per unit surface area and $\delta$ is the wall thickness. According to assumption ii), the rate of wall thinning due to in-plane expansion is matched exactly by deposition of new wall material. We therefore set $\dot{\epsilon}_{n}$ equal to zero.

\section{Equilibrium equations}

Since the wall of a growing cell yields slowly (small accelerations), we can assume that the forces acting on it are in equilibrium. For an axisymmetric tip, the meridional and circumferential

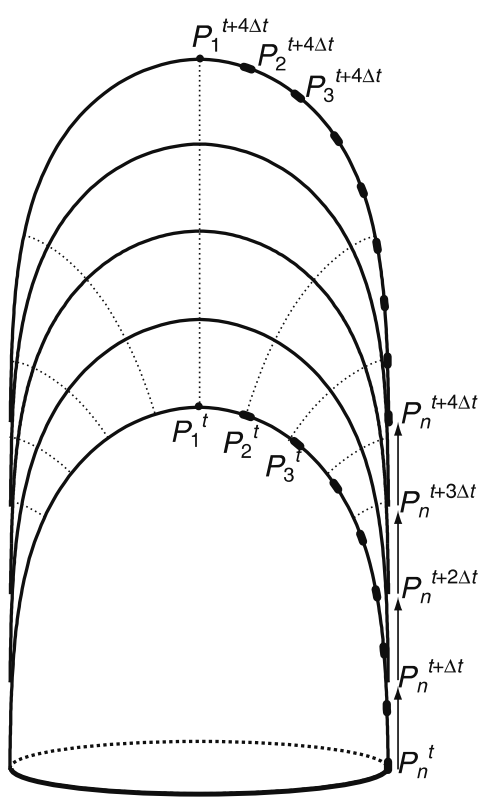

Fig. 6. Material point trajectories during steady-state growth. For simulations of tip growth, the cell meridian is discretized into $n$ points $P_{1}$ to $P_{n}$. The point denoting the equator $\left(P_{n}\right)$ is moved forward in steps to follow the advance of the tip.

stresses can be expressed directly as functions of pressure $(P)$ and the local geometry of the cell surface (see Appendix B):

$$
\begin{aligned}
\sigma_{s} & =\frac{P}{2 \delta \kappa_{\theta}} \\
\sigma_{\theta} & =\frac{P}{2 \delta \kappa_{\theta}}\left(2-\frac{\kappa_{s}}{\kappa_{\theta}}\right)
\end{aligned}
$$

The meridional and circumferential stresses are identical when $\kappa_{s}=\kappa_{\theta}$. This is the case at the pole of the cell where $\kappa_{s}$ and $\kappa_{\theta}$ converge to the same value. In the cylindrical portion of the cell where $\kappa_{s}$ is zero, the circumferential stress is twice the meridional stress. The ratio of stresses thus changes substantially along the cell meridian due to change in local curvature.

\section{Steady-state analysis}

As illustrated in Fig. 2, the three fundamental relations that describe cell morphogenesis constitute a feedback loop. Therefore, it is not a priorieasy to predict cell shape from wall mechanical properties or other parameters that might be under cellular control. We can gain some insight into this system of equations by considering the relation between wall mechanical properties and 
cell geometry at steady state and making use of some basic assumptions about the deformation process. One interesting choice for deformation is to assume that the displacement of material points is always perpendicular to the cell surface.

To show the type of solutions that can be achieved under the assumption made above, we must first define two ratios: the strain rate anisotropy $\lambda(s)=\left(\dot{\epsilon}_{\theta}-\dot{\epsilon}_{s}\right) /\left(\dot{\epsilon}_{\theta}+\dot{\epsilon}_{s}\right)$ and stress anisotropy $\gamma(s)=\left(\sigma_{\theta}-\sigma_{s}\right) /\left(\sigma_{\theta}+\sigma_{s}\right)$. Substituting these ratios in Eq. (5) and rearranging, we obtain to the following relation:

$$
\nu=\frac{\lambda-\gamma}{\lambda+\gamma}
$$

This important relation can be interpreted as an alternative definition for the flow coupling. Equation (11) illustrates an interesting parallel between viscoplasticity and linear elasticity since the same relation can be obtained for the Poisson's ratio as a function of the elastic strain anisotropy and the stress anisotropy. If the material is loaded in uniaxial stress, $\gamma$ is equal either 1 or 1 and Eq. (11) reduces to $\nu=-\dot{\epsilon}_{s} / \dot{\epsilon}_{\theta}$ or $\nu=-\dot{\epsilon}_{\theta} / \dot{\epsilon}_{s}$, the classical definition of the Poisson's ratio (Love, 1944).

Having derived Eq. (11), we can now return to the issue of finding analytical solutions under the assumption that the displacement of material points is perpendicular to the cell surface. Setting $v_{t}=0$ in Eqs. (6) and (7), we find that $\left.\lambda=\left(\kappa_{\theta}-\kappa_{s}\right) /\left(\kappa_{\theta}+\kappa_{s}\right)\right)$. Moreover, substitution of Eqs. (9) and (10) into the relation for the stress anisotropy yields $\gamma=\left(\kappa_{\theta}-\kappa_{s}\right) /\left(3 \kappa_{\theta}-\kappa_{s}\right)$. Substituting for $\lambda$ and $\gamma$ in Eq. (11) and rearranging, we obtain:

$$
\frac{\kappa_{s}}{\kappa_{\theta}}=1-2 \nu
$$

We have therefore been able to express cell geometry (given here by $\kappa_{s}$ and $\kappa_{\theta}$ ) directly in terms of one mechanical property (the flow coupling $\nu$ ). Equation (12) must satisfy three conditions in order to yield realistic tip shapes. At the pole $(s=0)$ the two curvatures must be equal thus $\nu(0)=0$. At the equator $(s=S)$, slope continuity between the dome and the cylindrical part of the cell requires that $d \kappa_{\theta} /\left.d s\right|_{S}=0$ or equivalently $\varphi(S)=\pi / 2$. Finally, to ensure continuity of curvature at the same location, $\nu(S)=1 / 2$ must be imposed so that $\kappa_{s} \rightarrow 0$ as $s \rightarrow S$. A discontinuity in the meridional curvature at the equator would lead to a stress discontinuity. Such discontinuities are rapidly removed in physical systems that undergo large deformations and, for the same reason, they are not likely to be present in walled cells.

Given a function $\nu=\nu(s)$, we look for a solution $\kappa_{s}(s)$ that satisfies both Eq. (12) and the boundary conditions. Note that because the tip is axisymmetric, $\kappa_{\theta}$ can be expressed in terms of $\kappa_{s}$ (see below). Solutions can be found iteratively by solving the following three equations in succession:

$$
\begin{aligned}
& \varphi(s)=\int_{0}^{s} \kappa_{s}^{(i)} d s \\
& \kappa_{\theta}^{(i)}=\sin \varphi r^{-1}=\sin \varphi\left(\int_{0}^{s} \cos \varphi(s) d s\right)^{-1} \\
& \kappa_{s}^{(i+1)}=(1-2 \nu) \kappa_{\theta}^{(i)}
\end{aligned}
$$

Here, $\kappa_{s}^{(i)}$ is the meridional curvature after $i$ iterations. We begin with an initial hemispherical tip geometry $\left(\kappa_{s}^{(0)}\right)$ with meridional arc length $S=1$. Solving Eq. (13) for the initial guess, we find a new tip geometry $\kappa_{s}^{(1)}$. If the geometry does not satisfy the boundary conditions at the outer margin (i.e. if $\varphi(S) \neq \pi / 2$ ) then the curvature is rescaled appropriately while the arc length is kept constant. After several iterations, this process converges to a tip geometry that is compatible both with the function selected for the flow coupling and with the boundary conditions. It must be emphasized that $\nu$ fixes the ratio of the two curvatures but not their
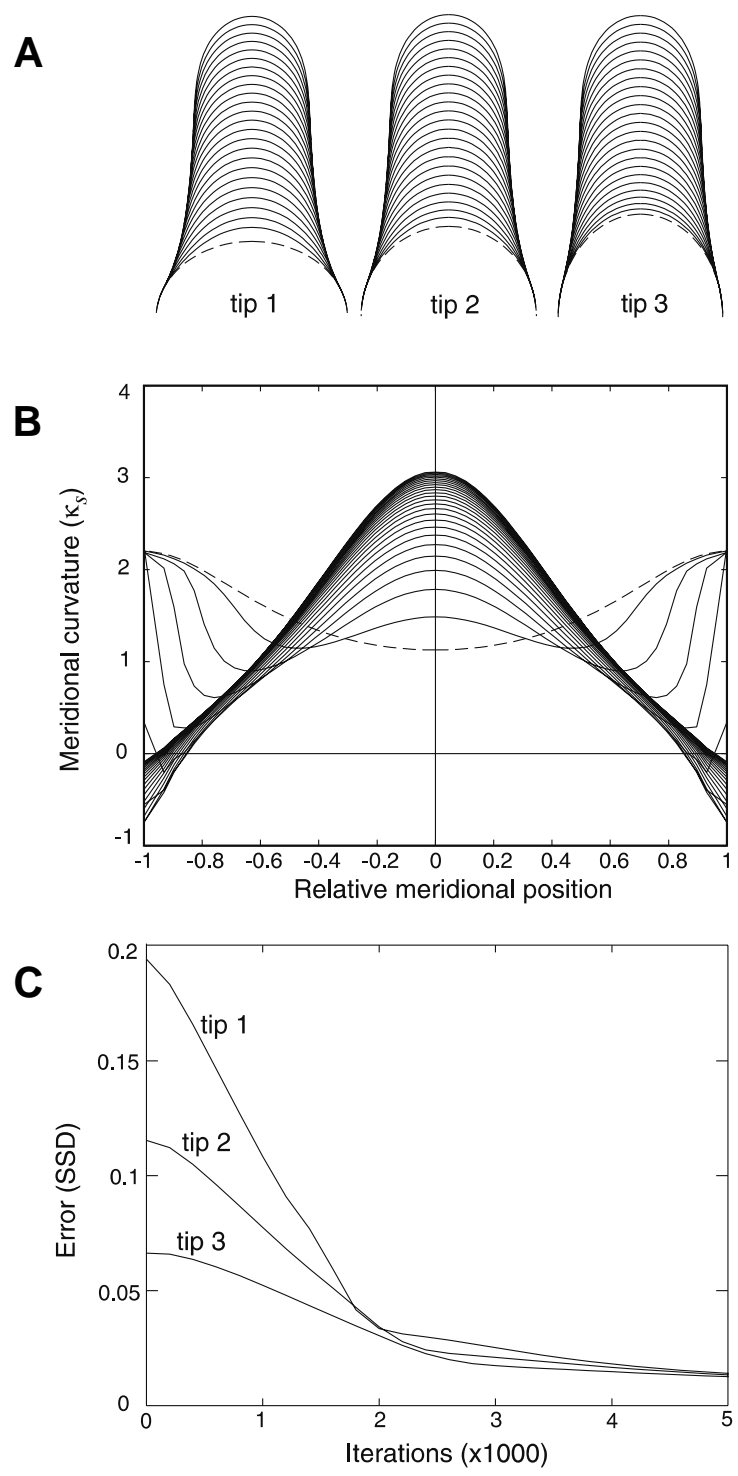

Fig. 7. Convergence to steady-state geometry. (A) Three tips with the same mechanical properties but different initial shapes. The evolution of the meridional curvature for tip 1 is given in (B) where the dashed line is the curvature for the initial geometry. (C) After a sufficient number of iterations, all tips converge to the predicted steady-state geometry. The error was measured as the sum of the squared differences (SSD) between the current curvature and the steady-state curvature derived analytically. 
absolute value; it thus defines the shape of the tip but not its size.

\section{Simulation of tip growth}

The great value of the normal displacement assumption is that it yields simple analytical solutions to an otherwise complicated system of equations. The assumption, however, is an idealized view of tip growth to which many exceptions are to be found. Deviations from normal displacement, even if these are small, will likely create conditions under which the flow coupling and other mechanical properties will share the control of cell shape. To study the general relation between wall mechanical properties and cell shape we must resort to computer simulations.

\section{Computational approach}

In our formulation of the constitutive relations, the rates of strain are determined by the current stress state and mechanical properties so that we can simulate large deformation of the cell surface as a series of small growth increments. The algorithm implemented with Matlab (The MathWorks) is composed of the following steps:

i) We first specify the initial tip geometry, turgor pressure and a set of mechanical properties. The initial, axisymmetric geometry of the tip is set by defining one of its meridians. The mechanical properties can be constant through time or vary.

ii) The cell's meridian is discretized with $n$ points $P_{i}=\left(x\left(s_{i}\right), y\left(s_{i}\right)\right)$ with $P_{1}$ lying at the pole of the tip and $P_{n}$ lying at the equator (Fig. 6). The arc position of point $P_{i}$ is denoted $s_{i}$. The local meridional curvature of the cell outline is determined by fitting a circle to three successive points $\left(P_{i-1}, P_{i}, P_{i+1}\right)$. The curvature at the middle point is the reciprocal of the circle's radius and is given by the equation: $\kappa_{s}^{i}=\left(2 \sin \rho_{i}\right) / l_{i}$, where $l_{i}$ is the straight distance between $P_{i-1}$ and $P_{i+1}$ and $\rho_{i}$ is the interior angle between the vectors joining $P_{i}$ to $P_{i-1}$ and $P_{i+1}$. Curvature at $P_{n}$ is extrapolated from the curvature at $P_{n-2}$ and $P_{n-1}$.

iii) Using the equilibrium equations (Eqs. (9),(10)), the stresses are computed from the current geometry and values for turgor pressure and wall thickness. Where the effective stress exceeds the yield stress, the meridional and circumferential stresses can be substituted in the constitutive equations (Eq. (5)) to get the strain rates.

iv) To determine the displacement velocities $\left(v_{n}\right.$ and $v_{t}$ ) from the strain rates, the kinematic relations must be inverted. Equations (6) and (7) can be combined and rearranged to give the following partial differential equation for $v_{t}$ :

$$
\frac{\partial v_{t}}{\partial s}-\frac{\kappa_{s} v_{t}}{\tan \varphi}=\dot{\epsilon}_{s}-\frac{\kappa_{s}}{\kappa_{\theta}} \dot{\epsilon}_{\theta}
$$

In this equation, all variables except $v_{t}$ are known functions of the meridional position, $s$. The boundary condition is $v_{t}(S)=0$, where $S$ is the meridional distance between the pole and the equator. The equation can be integrated to give $v_{t}$ :

$v_{t}(s)=\sin \varphi(s) \int_{S}^{s} \frac{1}{\sin \varphi(s)}\left(\dot{\epsilon}_{s}-\frac{\kappa_{s}}{\kappa_{\theta}} \dot{\epsilon}_{\theta}\right) d s$

By substitution into Eq. (7), a similar equation is found for $v_{n}$ :

$$
v_{n}(s)=\dot{\epsilon}_{\theta} / \kappa_{\theta}-\cos \varphi(s) \int_{S}^{s} \frac{1}{\sin \varphi(s)}\left(\dot{\epsilon}_{s}-\frac{\kappa_{s}}{\kappa_{\theta}} \dot{\epsilon}_{\theta}\right) d s
$$

v) The velocity of every point on the meridian is given by Eqs. (15) and (16). The new, deformed, geometry is determined by finding the position of each point after a small time interval, $\Delta t$. This process 'maps' the material point trajectories (Fig. 6). During a growth interval, some material points are displaced from the growing dome to the non-growing cylinder. These points are eliminated and replaced by new points within the growth region such that the length of the growth region and its spatial resolution are preserved. This is done by fitting the deformed meridian with a cubic spline and remeshing between $P_{1}$ and $P_{n}$ with a uniform spacing between points. Steps iito $v$ are repeated until a steady state is reached.

The convergence of the algorithm to a steady-state solution was checked against the analytical solutions derived in the previous section. Tips of different initial geometry but with the same arc length and mechanical properties were used (Fig. 7). Despite important differences in the initial curvature, the tips converged to the geometry that was analytically derived. These simulations indicate that there is a unique geometry corresponding to a given set of mechanical properties.

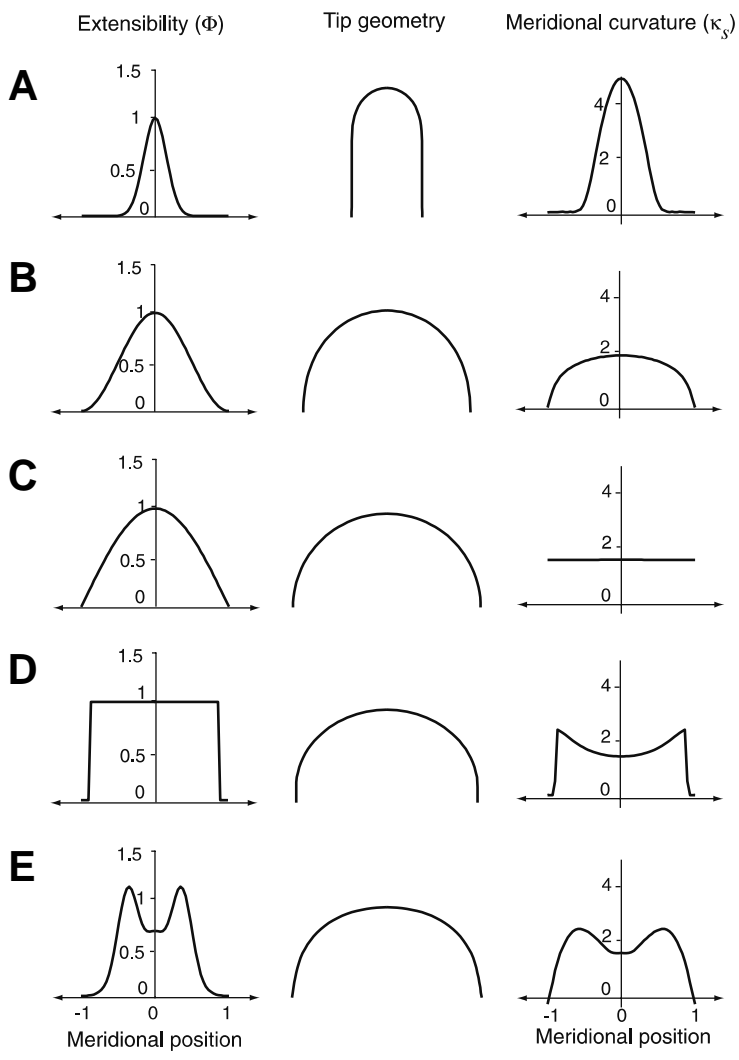

Fig. 8. Effect of wall extensibility $\Phi$ on the shape of the tip. In all cases the flow coupling was $\nu(s)=0.5$ and the yield stress $\left(\sigma_{y}\right)$ was constant and set to $50 \%$ of the minimal effective stress $\left(\sigma_{e}\right)$. 


\section{Role of mechanical properties in determining cell shape}

The role played by the mechanical properties in determining cell shape was investigated numerically. For brevity, only variations in $\Phi$ and $\nu$ will be considered here. Figures 8 and 9 show the tip geometry and meridional curvature for different combinations of $\Phi$ and $\nu$. Inspection of these figures reveals that tips with superficially similar geometries can nevertheless show substantial differences in their meridional curvatures. A basic constraint for the tip geometry is that the two flanks of the cell should be parallel at the equator. In terms of the meridional curvature, this geometrical constraint requires that the area under the curvature plot be equal to $\pi$. In other words, the total turning of the tip outline must be $\pi\left(180^{\circ}\right)$. Different tip geometries correspond to different distributions of the curvature. Prolate tips show high curvature near the pole while oblate tips show relatively more curvature away from the pole. The hemispherical tip geometry is an intermediate shape where curvature is constant along the meridian (Fig. $8 \mathrm{C})$.

The trend in Fig. 8 shows that a gradual shift of the extensibility away from the pole leads to a similar shift of the meridional curvature. In Fig. 9, raising the value of $\nu$ when it is constant along the meridian tends to make the distribution of curvature more uniform although the tip geometry is not very sensitive to such changes (Fig. 9A-C). On the other hand, if $\nu$ is graded then the effect is more pronounced. A gradual increase of $\nu$ from the pole

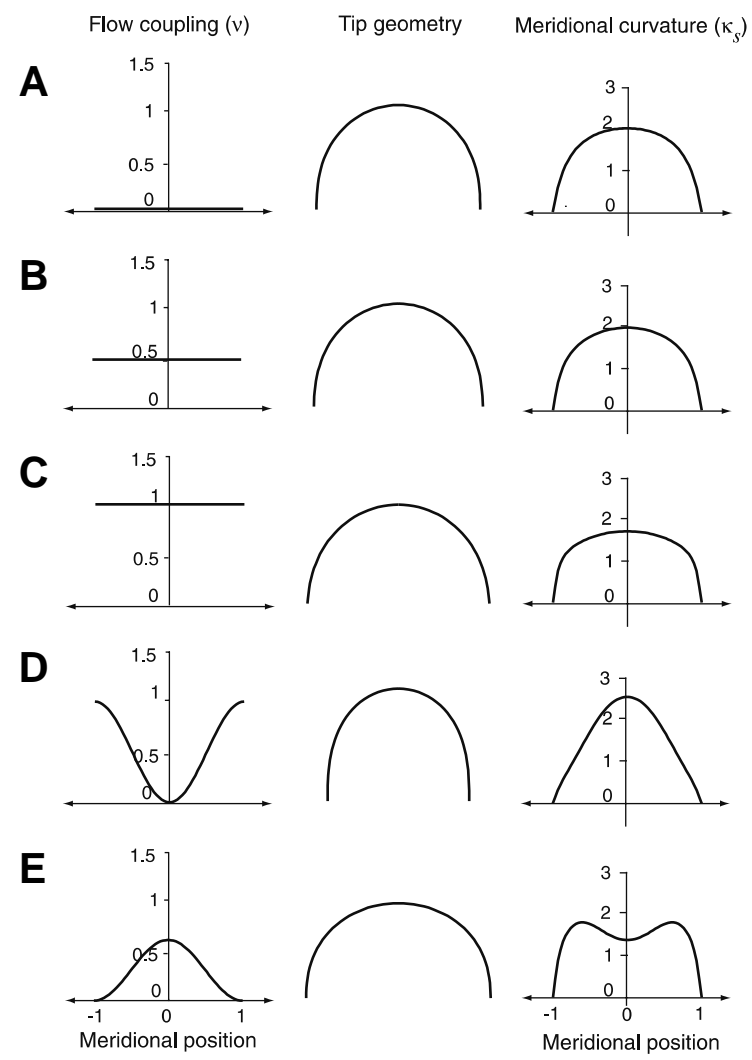

Fig. 9. Effect of flow coupling $\nu$ on the shape of the tip. In all cases the extensibility was $\Phi(s)=\cos (s \pi / 2)^{2}$ and the yield stress $\left(\sigma_{y}\right)$ was constant and set to $50 \%$ of the minimal effective stress $\left(\sigma_{e}\right)$.

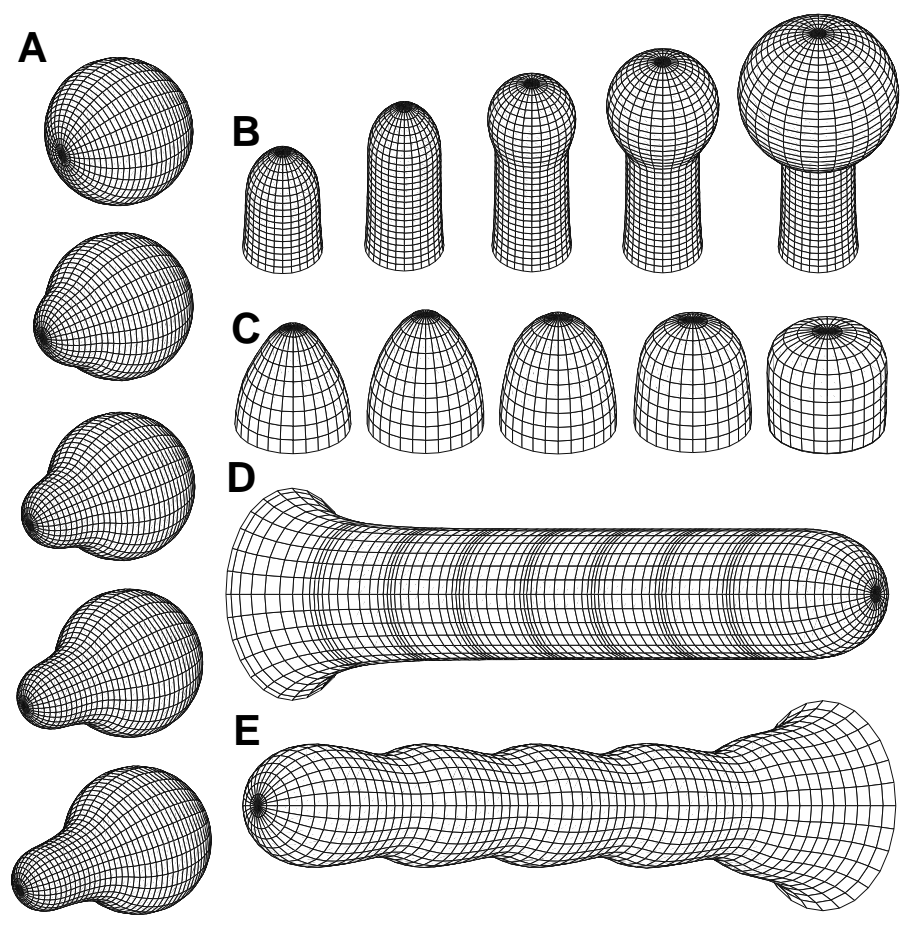

Fig. 10. Non-steady morphogenesis of cells. (A) Initiation of tip growth as in the Fucus egg. (B) Sporangium formation in Phycomyces. (C) Tip flattening as in the first phase of whorl initiation in Acetabularia. (D) Pulsatile growth in a tip-growing cell. Latitutinal lines show the fluctuations in the rate of advance of the tip. (E) Beaded shape in a tip-growing cell resulting from temporal fluctuations in the pattern of wall expansion.

to the equator favors a high curvature at the pole while a gradual decrease of $\nu$ favors a shift of curvature toward the equator (Fig. $9 \mathrm{D}$ and $\mathrm{E})$. We conclude that similar gradients of $\Phi$ and $\nu$ have opposite effects on the meridional curvature.

\section{Non-steady growth of cells}

Many phases of cell growth are not steady. In fact, one could argue that growth transients represent some of the most important events in morphogenesis. Examples include the initiation of tip growth from spherical spores or pollen grains and the formation of sporangia at the extremity of fungal hyphae. Given the significance of these morphogenetic events, it would be valuable to be able to model them. The simulation approach described above lends itself to this sort of problems.

Initiation of tip growth from roughly spherical or cylindrical cells is a common feature of pollen grains, fungal spores, trichoblasts and many algae, including the much-studied Fucus egg. This morphogenetic transition can be modeled by initiating tip growth on a sphere (Fig. 10A) without requiring any other change to the protocol already outlined. The reverse process of creating a spherical structure atop a long cylindrical cell is observed during the morphogenesis of the Phycomyces sporangiophore (Castle, 1942). The striking transition from tip growth to the formation of the spherical sporangium was simulated (Fig. 10B) by stopping the advance of the tip boundary (step vin the protocol) but leaving the mechanical properties unchanged. As a result, the tip starts to 


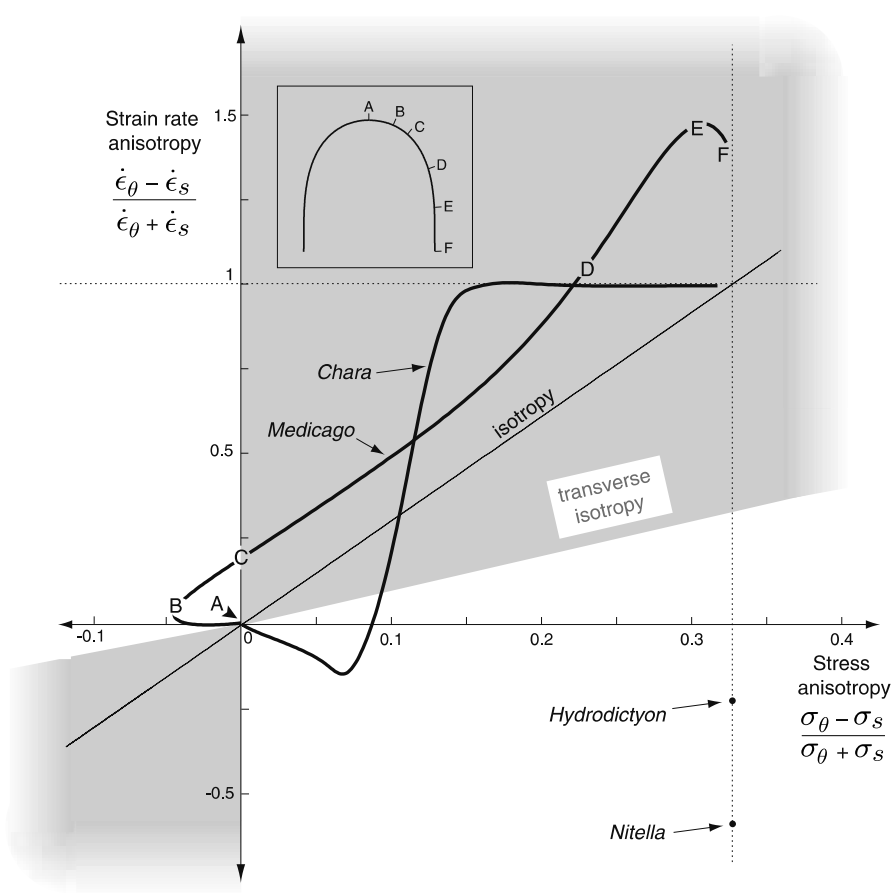

Fig. 11. Anisotropy space. Isotropic, transversely isotropic and anisotropic models span different subsets of that space. Models with isotropic mechanical properties are limited to a line passing through the origin (thin solid line). Models with transversely isotropic mechanical properties cover the shaded area in the anisotropy space. Finally, models with anisotropic mechanical properties extend to the whole space. Observed anisotropies for two tip-growing cells (Medicago truncatula root hair and the Chara rhizoid) and two diffusely-growing cells (Nitella and Hydrodictyon) are shown. The approximate location where the strain rate and stress anisotropies were recorded are shown for $\mathrm{M}$. truncatula (inset). For diffusively-growing cells such as Nitella and Hydrodictyon, wall expansion is relatively constant along the length of the cell. Therefore, only mean values are shown. A value of strain rate anisotropy above the horizontal dotted line indicates that the meridional strain rate is negative (contraction). The stress anisotropy for a tip-growing cell is expected to reach a maximum of $1 / 3$ (vertical dotted line) at the equator of the dome. Sources for the cell data used in this graph are given in the text.

increase in size and adopts a spherical shape.

In Acetabularia and other Dasycladalean algae, tip growth alternates with the formation of whorls of hairs. The first event in this transition is a characteristic flattening of the tip (Dumais and Harrison, 2000). Here the transition (Fig. 10C) was modeled by shifting the pattern of wall extensibility from one with a maximum at the pole to one with a maximum on the flank of the cell, as in Fig. $8 \mathrm{~A}$ and Fig. 8E, respectively. Numerous investigators have also reported fluctuations in the elongation rate of tip-growing cells (Holdaway-Clarke et al., 1997). In some instances the tip geometry remains unchanged but shows periodic bursts of growth (Fig. 10D) while in others, the tip geometry changes cyclically resulting in a beaded appearance of the cell shaft (Fig. 10E). The former mode of morphogenesis was simulated by rescaling a given wall extensibility profile over time and the latter mode was simulated by alternating between two distinct extensibility profiles.

Examples of most of the kinds of morphogenesis illustrated in Fig. 10 can be found both in normal fungal hyphal and conidial growth and, in exaggerated form, in fungal morphogenetic mu- tants (Springer and Yanofsky, 1989; Seiler and Plamann, 2003). Some of these mutants' effects are attributed to alterations of cytoskeletal or motor proteins (Xiang and Plamann, 2003). Presumably these morphogenetic effects result from modifications in either the rate, or the localization, of delivery to the apical dome's cell wall, of secreted agents (proteins, polysaccharides, $\mathrm{Ca}^{2+}$, etc.) that influence the mechanical properties of the wall.

\section{Anisotropy space}

Figures 8,9 and 10 show the range of shapes that can be achieved with a model that allows for spatial and temporal variations of mechanical properties. However, the geometries computed can be generated by a wide range of models (see for example Bartnicki-Garcia et al., 1989; and Goriely and Tabor, 2003) including models that assume an isotropic cell wall. One may therefore ask whether the inclusion of mechanical anisotropy in our model and the complications that follow are really necessary. The need for such a model is obvious if one considers the actual strain rates and stresses involved in cell morphogenesis. To show the importance of anisotropy, we rewrite Eq. 11 so that the strain rate anisotropy and the stress anisotropy are present only once:

$$
\lambda=\gamma \frac{1+\nu}{1-\nu}
$$

We now consider in general terms how the mechanical anisotropy - embodied by the flow coupling - can constrain the strain rates and stresses. For an isotropic material $(\nu=1 / 2)$, Eq. (17) gives $\lambda=3 \gamma$. Measured strain and stress anisotropies must therefore fall on a line of slope three for an isotropic material (Fig. 11). For a transversely isotropic material, the flow coupling can take values from the interval $[0,1]$. Substitution of the lower bound $\nu \geq 0$ and upper bound $\nu \leq 1$ in Eq. (17) leads to the following inequalities: $\lambda \geq \gamma$ if $\gamma>0, \lambda \leq \gamma$ if $\gamma<0$ and $\lambda=0$ if $\gamma=0$ (Fig. 11). Finally, for a fully anisotropic cell wall we must return to the general form of Eq. (5) and perform the same rearrangements that yielded Eq. (17). When this is done, it is found that strain anisotropy is not restricted by $\nu$ as given in Eq.
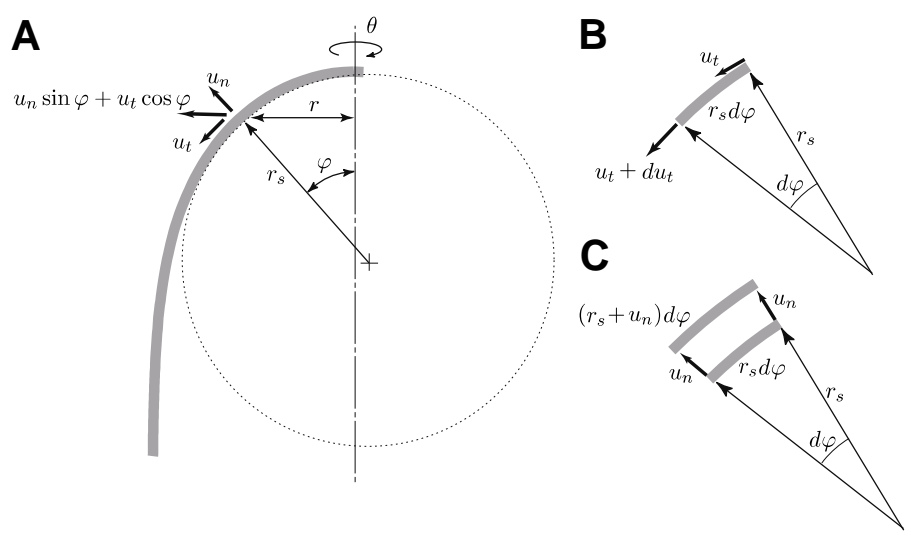

Fig. 12. Derivation of the kinematic relations. See Appendix A for details. 


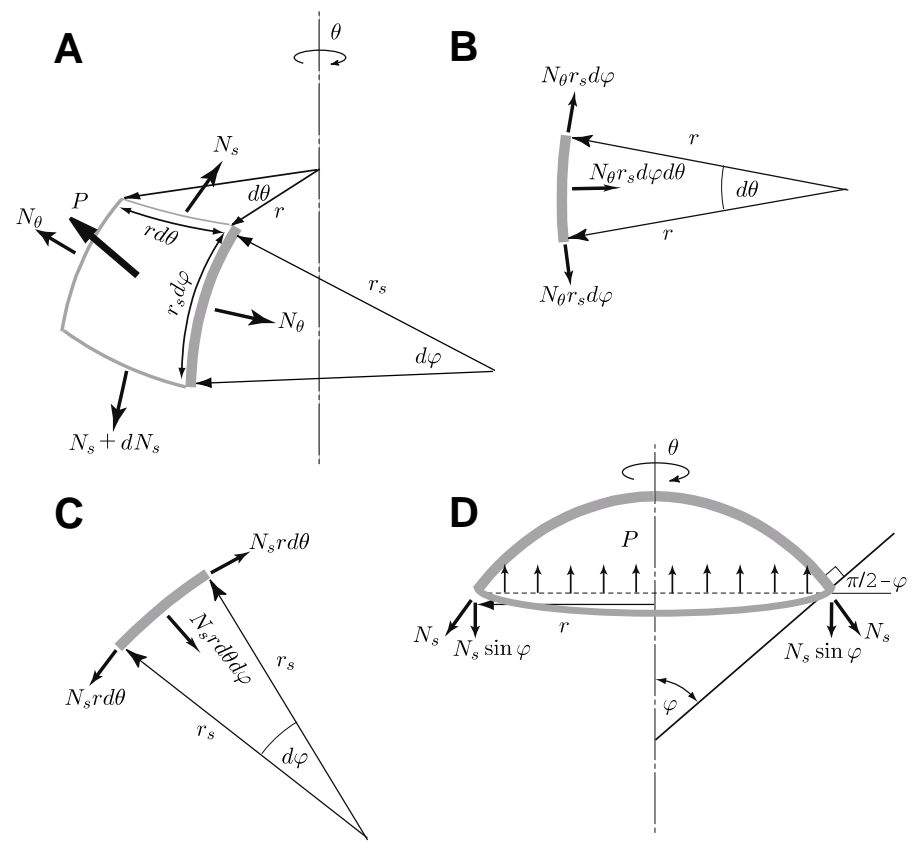

Fig. 13. Derivation of the equilibrium relations. See Appendix $B$ for details.

(17). In particular, $\lambda$ can differ from zero even when $\gamma=0$.

It is clear that the pattern of wall expansion is highly constrained for an isotropic material and less so for a material that is only transversely isotropic. We can ask whether observed patterns of strain rates in plant cells are such that they could be produced in a cell wall with isotropic mechanical properties. We have included in Fig. 11 the strain rate and stress anisotropies recorded for two tip-growing cells (root hair of Medicago truncatula, data from Dumais et al., 2004; the Chara rhizoid, data from Hejnowicz et al., 1977) and two diffusely-growing cells (the Nitella internodal cell and Hydrodictyon; data from Green, 1963). Nitella and Hydrodictyon lie clearly outside the range of strain and stress patterns accessible to an isotropic or transversely isotropic model. To be completely explained, morphogenesis in these cells thus requires fully anisotropic mechanical properties. Observations have demonstrated that indeed the cell wall of Nitella is highly anisotropic (Probine and Preston, 1962; Green, 1963). The strain rates and stresses observed in Medicago root hairs and the Chara rhizoid are distinct from those of diffusely-growing cells. For most of their ranges, the strain rate and stress anisotropies lie within the subspace accessible to a transversely isotropic model. The deviation near the origin suggests that a transversely isotropic model may not do full justice to tip growth. We have provided evidence elsewhere (Dumais et al., 2004) for a slight mechanical anisotropy in the plane of the cell wall although a simpler transversely isotropic cell wall can also account for the main features of tip growth, at least in root hairs of Medicago truncatula. The above observations show clearly that a fully isotropic cell wall cannot account for observed patterns of wall expansion in tip-growing cells and even less so in diffusely-growing cells.

\section{Conclusion}

We have presented a model for plant cell morphogenesis that offers a first attempt at integrating the mechanics of wall deformation with specific features of wall assembly. We provided a protocol to solve this model numerically and showed how it can be used to simulate different modes of morphogenesis (Fig. 10). As indicated in Fig. 2, the kernel of our model is a feedback loop linking wall turgor stresses, wall strain rates and cell geometry (see also Hejnowicz et al., 1977). According to this diagram, there is not a simple causal sequence linking the genes and cell shape. Although gene products and other regulatory molecules directly influence the mechanics of cell expansion, one cannot easily pinpoint their specific contributions to cell shape. This observation calls attention to the need to develop adequate models of cell morphogenesis concomitantly with traditional experimental approaches. In fact, our ability to simulate morphogenesis can play a critical role in supporting or extending our intuitions about the molecular and mechanical control of cell shape.

The model developed in this paper is based on the premise that plant cells control morphogenesis by modulating the viscoplastic properties of their wall. We give here an analysis of wall strains and stresses reported in the literature, which indicates that some anisotropy must be built into models to account for observed patterns of wall expansion (Fig. 11). We have tackled this problem by taking into account the mechanical anisotropy that can arise from different organizations of cellulose microfibrils in the cell wall. However, various other molecules such as expansins (McQueen-Mason and Cosgrove, 1995; Cosgrove, 2000), xyloglucan endotransglycosylase (Antosiewicz et al., 1997; Campbell and Braam, 1999), xyloglucan hydrolase (Kaku et al., 2002) and yieldins (Okamoto-Nakazato et al., 2000a,b) are also known to alter the wall mechanical properties although the physical directionality of the effects has not been determined. To be biophysically persuasive, the action of the wall-modifying molecules, as well as any model of wall expansion that is based on biosynthetic addition of new material (Bartnicki-Garcia et al., 1989; Gierz and Bartnicki-Garcia, 2001), must include features that affect the directionality of cell wall yield in a manner that brings about the morphogenesis that is observable during cell growth. We hope that this work, by offering an explicit biophysical model of tip-growth morphogenesis, will provide a useful starting point for addressing these issues.

We thank Drs. Dorota Kwiatkowska, Richard Christensen, David Ackerly, Enrique Cerda and Joseph Keller for comments on an earlier version of this manuscript. JD acknowledges support from the FCAR fund (Québec), the Center for Computational Genetics and Biological Modeling (Stanford University) and a research fellowship from Prof. L. Mahadevan (University of Cambridge).

\section{References}

ANTOSIEWICZ, D.M., PURUGGANAN, M.M., POLISENSKY, D.H. and BRAAM, J. (1997) Cellular localization of Arabidopsis xyloglucan endotransglycosylaserelated proteins during development and after wind stimulation. Plant Physiol. 115: 1319-1328.

BARTNICKI-GARCIA, S., HERGERT, F., and GIERZ, G. (1989) Computer simula- 
tion of fungal morphogenesis and the mathematical basis for hyphal (tip) growth. Protoplasma 153: 46-57.

BASKIN, T.I. (2001) On the alignment of cellulose microfibrils by cortical microtubules: a review and a model. Protoplasma 215: 150-171.

BELFORD, D.S. and PRESTON, R.D. (1961) The structure and growth of root hairs. J. Exp. Bot. 12: 157-168.

BINGHAM, E.C. (1922) Fluidity and Plasticity. McGraw-Hill, New York.

CAMPBELL, P. and BRAAM, J. (1999) In vitro activities of four xyloglucan endotransglycosylases from Arabidopsis. Plant J. 18: 371-382.

CAPSONI, A., CORRADI, L. and VENA, P. (2001) Limit analysis of orthotropic structures based on Hill's yield condition. Int. J. Solids Struc. 38: 3945-3963.

CASTLE, E.S. (1942) Spiral growth and reversal of spiraling in Phycomyces, and their bearing on primary wall structure. Amer. J. Bot. 29: 664-672.

CASTLE, E.S. (1955) The mode of growth of epidermal cells of the Avena coleoptile. Proc. Nat. Acad. Sci. USA 41: 197-199.

CASTLE, E.S. (1958) The topography of tip growth in a plant cell. J. Gen. Physiol. 41: 931-926.

CHAPLAIN, M.A.J. and SLEEMAN, B.D. (1990) An application of membrane theory to tip morphogenesis in Acetabularia. J. Theor. Biol. 146: 177-200.

CHEN, J.C.W. (1973) The kinetics of tip growth in the Nitella rhizoid. Plant Cell Physiol. 14: 631-640.

CHRISTENSEN, R.M. (1997) Yield functions/failure criteria for isotropic materials. Proc. Roy. Soc. Lond., Series A 453: 1473-1491.

CLELAND, R. (1959) Effect of osmotic concentration on auxin-action and on irreversible and reversible expansion of the Avena coleoptile. Physiol. Planta. 12: 809-825.

COSGROVE, D.J. (1985) Cell wall yield properties of growing tissue: Evaluation by in vivo stress relaxation. Plant Physiol. 78: 347-356.

COSGROVE, D.J. (2000) Loosening of plant cell walls by expansins. Nature 407: 321-326.

DA RIVA RICCI, D. and KENDRICK, B. (1972) Computer modelling of hyphal tip growth in fungi. Can. J. Bot. 50: 2455-2462.

DUMAIS, J. and HARRISON, L.G. (2000) Whorl morphogenesis in the dasycladalean algae: the pattern formation viewpoint. Phil. Trans. R. Soc. London 355: 281305.

DUMAIS, J., LONG, S.R. and SHAW, S.L. (2004) The mechanics of surface expansion anisotropy in Medicago truncatula root hairs. Plant Physiol. 136: 3266-3275.

FLUGGE, W. (1973) Stresses in Shells. Springer-Verlag, Berlin.

GIERZ, G. and BARTNICKI-GARCIA, S. (2001) A three-dimensional model of fungal morphogenesis based on the vesicle supply center concept. $J$. Theor. Biol. 208: 151-164.

GORIELY, A. and TABOR, M. (2003) Biomechanical models of hyphal growth in actinomycetes. J. Theor. Biol. 222: 211-218.

GREEN, P.B. (1962) Mechanism for plant cellular morphogenesis. Science 138: 1404-1405.

GREEN, P.B. (1963) Cell walls and the geometry of plant growth. InMeristems and Differentiation. Brookhaven Symposia in Biology 16: 203-217.

GREEN, P.B., ERICKSON, R.O. and BUGGY, J. (1971) Metabolic and physical control of cell elongation rate. In vivo studies in Nitella. Plant Physiol. 47: 423430.

HABERLANDT, G. (1887) Uber die Beziehungen zwischen Function und Lage des Zellkernes bei den Pflanzen. Fischer, Jena.

HART, T.N. and TRAINOR, L.E.H. (1989) Geometrical aspects of surface morphogenesis. J. Theor. Biol. 138: 271-296.

HEATH, I.B. ed. (1990) Tip Growth in Plant and Fungal Cells. Academic Press, San Diego.

HEJNOWICZ, Z., HEINEMANN, B. and SIEVERS, A. (1977) Tip growth: Patterns of growth rate and stress in the Chara rhizoid. Z. Pflanzenphys. 81: 409-424.

HEJNOWICZ, Z. and SIEVERS, A. (1971) Mathematical model of geotropically bending Chara rhizoids. Z. Pflanzenphys. 66: 34-48.

HILL, R. (1950) The Mathematical Theory of Plasticity. Oxford University Press, Oxford.
HOHENEMSER, K. and PRAGER, W. (1932) Uber die Ansatze der Mechanik isotroper Kontinua. Z. ang. Math. Mech. 12: 216-226.

HOLDAWAY-CLARKE, T.L., FEIJ O, J.A., HACKETT, G.R., KUNKEL, J.G. and HEPLER, P.K. (1997) Pollen tube growth and intracellular cytosolic calcium gradient oscillate in phase while extracellular calcium influx is delayed. PlantJ. 9: 1999-2010.

HOUWINK, A.L. and ROELOFSEN, P.A. (1954) Fibrillar architecture of growing plant cell walls. Acta Bot. Neerl. 3: 385-395.

KAKU, T., TABUCHI, A., WAKABAYASHI, K., KAMISAKA, S. and HOSON, T. (2002) Action of xyloglucan hydrolase within the native cell wall architecture and its effect on cell wall extensibility in azuki bean epicotyls. Plant Cell Physiol. 43: 21-26.

KATAOKA, H. (1982) Colchicine-induced expansion of Vaucheria cell apex. Alteration from isotropic to transversally anisotropic growth. Bot. Mag. 95: 317-330.

KELKAR, V.S. and SEWELL, R.T. (1987) Fundamentals of the Analysis and Design of Shell Structures. Prentice-Hall, Englewood Cliffs.

KIERMAYER, O. (1964) Untersuchungen über die Morphogenese und Zellwandbildung bei Micrasterias denticulata Bréb. Protoplasma 59: 76-132.

LOCKHART, J.A. (1965a) An analysis of irreversible plant cell elongation. J. Theor. Biol. 8: 264-275.

LOCKHART, J.A. (1965b) Cell extension. In Plant Biochemistry. BONNER, J. and VARNER, J.E. editors. Academic Press, New York.

MARTYNOV, L.A. (1975) A morphogenetic mechanism involving instability of initial form. J. Theor. Biol. 52: 471-480.

MCQUEEN-MASON, S.J. and COSGROVE, D.J. (1995) Expansin mode of action on cell walls. Analysis of wall hydrolysis, stress relaxation, and binding. Plant Physiol. 107: 87-100.

METRAUX, J.P., RICHMOND and P.A., TAIZ, L. (1980) Control of cell elongation in Nitella axillaris by endogenous cell wall pH gradients: Multiaxial extensibility and growth studies. Plant Physiol. 65: 204-210.

MONEY, N.P. and HAROLD, F.M. (1992) Extension growth of the water mold Achlya: Interplay of turgor and wall strength. Proc. Natl. Acad. Sci. USA 89: 4245-4249.

NEWCOMB, E.H. and BONNETT, H.T. (1965) Cytoplasmic microtubule and wall microfibril orientation in root hairs of radish. J. Cell Biol. 27: 575-589.

OKAMOTO-NAKAZATO, A., NAKAMURA, T. and OKAMOTO, H. (2000a) The isolation of wall-bound proteins regulating yield threshold tension in glycerinated hollow cylinders of cowpea hypocotyl. Plant Cell Env. 23: 145-154.

OKAMOTO-NAKAZATO, A., TAKAHASHI, K., KIDO, N., OWARIBE, K. and KATOU, K. (2000b) Molecular cloning of yieldins regulating the yield threshold of cowpea cell walls: cDNA cloning and characterization of recombinant yieldin. Plant Cell Env. 23: 155-164.

OLDROYD, J.G. (1947) A rational formulation of the equations of plastic flow for a Bingham solid. Proc. Camb. Phil. Soc. 43: 100-105.

ORTEGA, J.K.E. (1990) Governing equations for plant cell growth. Physiol. Planta. 79: 116-121.

PASSIOURA, J.B. and FRY, S.C. (1992) Turgor and cell expansion: Beyond the Lockhart equation. Aust. J. Plant Physiol. 19: 565-576.

PELCÉ, P. and POCHEAU, A. (1992) Geometrical approach to the morphogenesis of unicellular algae. J. Theor. Biol. 156: 197-214.

PRAGER, W. (1937) Mecanique des solides isotropes au delà du domaine elastique. Mem. Sci. Math. 87: 1-66.

PRAGER, W. (1961) Introduction to Mechanics of Continua. Ginn and Company, Boston.

PROBINE, M.C. (1966) The structure and plastic properties of the cell wall of Nitella in relation to extension growth. Aust. J. Biol. Sci. 19: 439-457.

PROBINE, M.C. and PRESTON, R.D. (1962) Cell growth and the structure and mechanical properties of the wall in internodal cells of Nitella opaca.ll. Mechanical properties of the walls. J. Exp. Bot.13: 111-127.

PROSEUS, T.E., ORTEGA and J.K.E., BOYER, J.S. (1999) Separating growth from elastic deformation during cell enlargement. Plant Physiol. 119: 775-784.

REINHARDT, M.O. (1892) Das Wachsthum der Pilzhyphen. Jahrb. wiss. Bot. 23 : 479-566.

RICHMOND, P.A., METRAUX, J.P. and TAIZ, L. (1980) Cell expansion patterns 
and directionality of wall mechanical properties in Nitella axillaris. Plant Physiol. 65: 211-217.

ROY, S.J., HOLDAWAY-CLARKE, T.L., HACKETT, G.R., KUNKEL, J.G., LORD, E.M. and HEPLER, P.K. (1999) Uncoupling secretion and tip growth in lily pollen tubes: evidence for the role of calcium in exocytosis. Plant J. 19: 379-386.

SCHROTER, K. and SIEVERS, A. (1971) Effect of turgor reduction on the Golgi apparatus and cell wall formation in root hairs. Protoplasma 72: 203-211.

SEILER, S. and PLAMANN, M. (2003) The genetic basis of cellular morphogenesis in the filamentous fungus Neurospora crassa. Mol. Biol. Cel/14: 4352-4364.

SHAW, S.L., DUMAIS, J. and LONG, S.R. (2000) Cell surface expansion in polarly growing root hairs of Medicago truncatula. Plant Physiol. 124: 959-969.

SPRINGER, M.L. and YANOFSKY, C. (1989) A morphological and genetic analysis of conidiophore development in Neurospora crassa. Genes Dev. 3: 559-571.
STEELE, C.R. (2000) Shell stability related to pattern formation in plants. J. Appl. Mech. 67: 237-247.

UGURAL, A.C. (1999) Stresses in Plates and Shells. McGraw-Hill, Boston.

UGURAL, A.C. and FENSTER, S.K. (1975) Advanced Strength and Applied Elasticity. American Elsevier, New York.

VON DASSOW, M., ODELL, G. and MANDOLI, D. (2001) Relationships between growth, morphology and wall stress in the stalk of Acetabularia acetabulum. Planta 213: 659-666.

XIANG, X. and PLAMANN, M. (2003) Cytoskeleton and motor proteins in filamentous fungi. Curr. Opin. Microbiol. 6: 628-633.

ZHU, G.L. and BOYER, J.S. (1992) Enlargement in Chara studied with a turgor clamp: Growth rate is not determined by turgor. Plant Physiol. 100: 2071-2080.

\section{Appendix A}

\section{Derivation of kinematic relations}

Our derivation of the kinematic relations follows the standard engineering derivation (Kelkar and Sewell, 1987; Ugural, 1999). In the derivation that follows the deformation of the surface is assumed to be infinitesimal. Consider a small meridional element (Fig. 12). The segment length $(d s)$ is given by its radius of curvature $\left(r_{s}\right)$ times the arc angle $(d \varphi)$ defined by the segment. After a small time interval $d t$, the segment is displaced in the tangential and normal directions by $u_{t}$ and $u_{n}$, respectively. The deformation of the segment may be regarded as composed of an increase in length $\left(d u_{t} / d \varphi\right) d \varphi$, owing to the gradient in tangential displacement and an increase in length $u_{n} d \varphi$ resulting from the radial displacement (Figure 12B,C). Dividing by the initial length of the segment $\left(d s=r_{s} d \varphi\right)$, we obtain the relative meridional extension (meridional strain):

$$
\epsilon_{s}=\frac{1}{r_{s}} \frac{d u_{t}}{d \varphi}+\frac{u_{n}}{r_{s}}=\frac{d u_{t}}{d s}+\kappa_{s} u_{n}
$$

where the relation $\kappa_{s}=1 / r_{s}=d \varphi / d s$ was used. The circumferential strain is equal to the increase in circumference $2 \pi\left(u_{n} \sin \varphi+u_{t} \cos \varphi\right)$ divided by the initial circumference $2 \pi r$
(Fig. 12A):

$$
\epsilon_{\theta}=\frac{u_{n} \sin \varphi+u_{t} \cos \varphi}{r}
$$

The strain rates $(\dot{\epsilon})$ are obtained from Eqs. (A.1), (A.2) by noting that for a short time interval $d t$, the displacements $u_{t}$ and $u_{n}$ equal $v_{t} d t$ and $v_{n} d t$ respectively, where $v_{t}$ and $v_{n}$ are displacement velocities. Equations (A.1) and (A.2) become:

$$
\begin{aligned}
\dot{\epsilon}_{s} & =\frac{\partial v_{t}}{\partial s}+\kappa_{s} v_{n} \\
\dot{\epsilon}_{\theta} & =\frac{v_{n} \sin \varphi+v_{t} \cos \varphi}{r}
\end{aligned}
$$

A partial derivative is used to emphasize that the velocity of material points depends not only on their meridional position $(s)$ but can also depend on time in non-steady morphogenetic phenomena like those shown in Fig. 10.

\section{Appendix B}

\section{Derivation of equilibrium equations}

Derivation of the equilibrium equations is done in terms of forces and stress resultants (Kelkar and Sewell, 1987; Ugural, 1999). A stress resultant is a force distributed over a length and corresponds to the stress integrated through the cell wall thickness. For an axisymmetric cell geometry loaded with turgor pressure $(P)$ two stress resultants, $N_{s}$ and $N_{\theta}$, need to be considered. The forces represented by the stress resultants and turgor pressure must be in equilibrium in all directions. Starting with the direction normal to the cell surface, the force exerted by turgor pressure is equal to $P(r d \theta)\left(r_{s} d \varphi\right)$ where $(r d \theta)\left(r_{s} d \varphi\right)$ is the area of the shell element (Fig. 13A). The forces acting on the upper and lower edges are equal to $N_{s} r d \theta$ (neglecting higher order terms). The component of the meridional force acting in the normal direction is equal to $2 N_{s} r d \theta \sin (d \varphi / 2)$ which, owing to the fact that $d \varphi$ is small, can be approximated as $N_{s} r d \theta d \varphi$ (Fig. 13C). Similarly, the forces acting on the side edges are equal to $N_{\theta} r_{s} d \varphi$. The component of the circumferential force pointing in the radial direction is $N_{\theta} r_{s} d \varphi d \theta$ (Fig. 13B). Finally, the component of that force acting normal to the cell surface is:

$N_{\theta} r_{s} d \varphi d \theta \cos (\pi / 2-\varphi)=N_{\theta} r_{s} d \varphi d \theta \sin \varphi$, where $\pi / 2-\varphi$ is the angle between the normal to the surface and the radial direction (Fig. 13D). 
Equilibrium in the normal direction requires that:

$N_{s} r d \theta d \varphi+N_{\theta} r_{s} d \varphi d \theta \sin \varphi=P(r d \theta)\left(r_{s} d \varphi\right)$

dividing by the area $r r_{s} d \varphi d \theta$ and using the identity $r_{\theta}=r / \sin \varphi$ (see Fig. 4) we get:

$$
\frac{N_{s}}{r_{s}}+\frac{N_{\theta}}{r_{\theta}}=P
$$

Next we determine the force equilibrium in the axial direction for a section of the cell intercepted by an angle $\varphi$ (Fig. 13D). The axial force exerted by turgor pressure is equal to $P \pi r^{2}$ while the axial force due to the meridional stress resultant is equal to $N_{s} 2 \pi r \sin \varphi$. Equilibrium requires that these two forces are of the same magnitude. Rearranging for $N_{s}$, the axial equilibrium equation is:

$$
N_{s}=\frac{P r}{2 \sin \varphi}=\frac{P r_{\theta}}{2}
$$

Substituting Eq. (B.3) into Eq. (B.2) gives the following expression for $N_{\theta}$ :

$$
N_{\theta}=\frac{P r_{\theta}}{2}\left(2-\frac{r_{\theta}}{r_{s}}\right)
$$

The meridional and circumferential stresses are obtained by dividing Eqs. (B.3) and (B.4) by the wall thickness $(\delta)$ :

$$
\sigma_{s}=\frac{\operatorname{Pr}_{\theta}}{2 \delta}=\frac{P}{2 \delta \kappa_{\theta}}
$$

$$
\sigma_{\theta}=\frac{P r_{\theta}}{2 \delta}\left(2-\frac{r_{\theta}}{r_{s}}\right)=\frac{P}{2 \delta \kappa_{\theta}}\left(2-\frac{\kappa_{s}}{\kappa_{\theta}}\right)
$$

